\title{
Be True: A Theory on Bilateral Sway
}

\author{
Emmet McManus* \\ *Corresponding author: Emmet McManus; Email: emmetmcmanus@hotmail.com
}

Received: December 11, 2021; Accepted: December 19, 2021; Published: December 31, 2021

\section{Introduction}

"The quest for truth is hard, but in some ways, it's also easy" Aristotle.

This theory attempts to identify and pinpoint exactly how and when many cancers, mental illnesses and other conditions may begin and unfold (tbd). Once understood, only then will we understand this information is intended for the attention of those of us who care. Is this theory a novel way to consider and hence a probable, fact-based and pragmatic explanation about what some of us are searching for, in an attempt to resolve and gain a true understanding of many of those mental and physical health disorders and problems (which they are not; they are difficulties and challenges)?

By what process are each other's personal 'functional bilateral cohesive synthesis' profiles determined? (The whole working quality of both sides. L/R) Does the outcome impact on and reflects in genetic regulation and functional mechanisms in each of our 11 body systems?

Effectively, would exploring the two fundamental questions suggested within this theory, further our understanding to help 'balance the sway' of what is arguably the largest unaddressed thorn in societies side today which, until purposefully investigated and hopefully appreciated, shall simply not go away?

This theory seeks to highlight a new alternative view on the possible origins of many individual neurological and physiological cases, and how they 'become' and develop. Then it queries: aren't they natural? Is a chiral (conflict/accord) type effect involved? Are named labels misleadingly?

Realistically, could it be that bilateral sway associates with health \& wellbeing on a comparably similar scale that $\mathrm{e}=\mathrm{mc2}$ associates with physics? Are there any limits or boundaries? (Self-esteem; Family; Education; Employment; Societal; Justice; Economy; Equality; Righteousness.)

Under what circumstances can this 'balance of sway' be considered to be 'tipped' ( $\mathrm{L}$ or R) and could it be manipulated? What are the implications of Bilateral Sway? Please peruse, and critique where necessary, this theory \& if you find it interesting, howsoever, share your views please.

Each on our own does not have the ability to work everything out, but we ALL may have something useful to say or indeed ASK! Initial feedback on this theory is indicative of 'a unique perspective'/'very intriguing'/'needs to be investigated further'
N.B. There are 10 questions being asked above, all of which need answering!

To whomever it may concern (i.e. everyone), a 'functional bilateral cohesive synthesis' profile (the whole working quality of both sides) - which we each uniquely possess - may help to better explain, if understood, the multitude of disorders and dysfunctions, as well as talents and skills, being experienced today; whether neurological or phycological (particular emphasis being on many mental health conditions \& diseases). ${ }^{\star}$ May help with: 'Scientists have some idea, however........ haven't worked out a cure yet'.

BUT HOW? Bilaterality is amongst the first, the most consistent and the most successful of all evolutionary trends that evolved around 575 million years ago.

Though bilateral, we are NOT mirror images of ourselves and the significance of this, however subtle, may be somewhat overlooked with respect to location \& impact.

Starting from cell structure upwards, no two paired organs for example, within our bodies function/perform equally, resulting in a dominant/recessive partnership. Within our genetic makeup, the scale and scope of every possibility must truly be immense ('Genetic sway').

Examples worth noting are L/R hemispheres, eye dominance, nasal cycle and also handedness - some of the more obvious sensory functions. But what about all the other organs and functions within our body systems? And there are many, including the gonads!

Ideally, this 'whole bilateral profile' wants to be well balanced/ homeostatic - no friction, no mutant behaviour. But as things are rarely just black and white, fluctuating shades of grey may appear throughout, indicating imbalances to varying degrees.

There is simply too much to get into \& too many questions at this point, so please if I may, allow me to cut to and offer a concluding view for consideration in order to elicit some thoughts on this theory. (Maybe even some assistance/feedback if that is an option.)

Ultimately, and with respect to the five points above, many conditions arising - most notably in mental health - could be better appreciated \& may actually be able to be pinpointed by enquiring and truly understanding the meaning behind the answers to two straight forward questions.

We each were created at the moment of conception from of a single sperm and a single egg. Sperm from the testes and an egg from the ovaries. This does not make sense: 1 sperm from both testes (L/R) 
and 1 egg from two oscillating ovaries (L/R). So how does that work then?

Understanding that paired organ partnerships can exhibit dominant or recessive traits (function) and hence could be considered bilaterally sensitive or influenced (performance), how does this manifest and what could the implications be?

\section{Manifest}

On a punnet square: L/L; L/R; R/L; R/R. (50:50 ratio)

i.e. 50 Bilateral; 50 Functional. Both are preferable, but only 1 possible. Ref: "Rule of thumb" experiment mentioned above.

Science generally implies that opposites are complimentary. Therefore $\mathrm{L} / \mathrm{R} \& \mathrm{R} / \mathrm{L}$ would appear compatible and want to function, though at the expense of Bilaterality. (50)

Conversely, Science also generally implies that likes are not complimentary. Therefore R/R \& L/L would appear incompatible and not want to function, though reinforcing Bilaterality. (50)

Since no known mechanism for guiding contralateral/ipsilateral fertilisation exists, we most probably 'could have been' or 'will be' hoping and wishing for things to work out well, which is good, though being unaware of a notable, fluctuating 2:1 probability ratio attached. (Science, it seems, says nothing about this!) A very important point, nevertheless, is that all hopes and wishes invariably seem somehow honoured with a beautiful baby.

\section{Implications}

On a general empirical spectrum, and being conscious to avoid confusing the stereotypical 'academic bank robber' with the 'school dropout surgeon' ;) - from a child that appears [naturally gifted/talented and may only struggle a little] to a child that appears to be [struggling somewhat, possibly, 'for unexplained reasons', yet tries hard] the possibilities seem endless, though currently stands at around 7.8 billion, or thereabouts, albeit the probabilities remain 2:1. These children become adult men and women \& these adults can be seen everywhere from our churches, through to our jails and that's neurological! For physiological viewing, you need to go from our churches, through to our hospitals \& sadly sometimes back to the church.

\section{SO!}

The two questions, rhetorically speaking, essentially are:

1-Which paternal gonad (testi) produced the gamete (sperm) that helped create you? (L or R)

2-Which maternal gonad (ovary) produced the gamete (egg) that helped create you? (L or R)

Parental bilateral genetic profiles, were they to be known, would prove intriguing. Obviously, it is impossible to know what the answers to these questions are and has been throughout our ancestral history, though this doesn't make it any less true or relevant when trying to understand the causes and reasons behind, and possibly an explanation for, both mental (neurological) and functional (physiological) conditions (good and not so good!).
Once created at conception, this unique bilateral genetic profile now begins to establish and then fulfil its role in 'instructing' how we individually will naturally function. The subsequent neurological, physiological, psychological, nutritional and environmental factors, which are all every bit as important, will start to play their respective roles in becoming decisive at 'influencing' how we each developmentally perform. We all carry two copies of each gene - alleles (outside of male sex chromosomes) - one from each parent. Very little, if anything, is known about differences, however subtle, within our DNA (genetic variation) from a bilateral sway perspective and its effects within out systems. This bilateral phenomenon influencing our DNA may be the main mechanism behind determining how genes are expressed along with chemical and molecular level factors. Varying abilities and/or disabilities, both neurological and physiological, could be the net result depending on how harmoniously balanced this new profile is. The potential contralateral/ipsilateral connectivity range encompassing both the dominant/recessive and L/R aspects (Functional \& Bilateral) and resulting in 16 different possibilities is fundamental to understanding the concept of this theory. Half of these 16 variants could be what are responsible for causing many mutations! It may also explain why mutations (maybe excluding random) can be either beneficial or harmful (good \& not so good!). Bilateral Sway may help to better explain Genetic Drift, which is described as a change in the gene pool that takes place strictly by change, resulting in genetic traits becoming lost or widespread in populations without respect to the survival or reproductive value of the alleles involved; also possibly resolving the "may or may not run in families" contradiction. This unquantifiable matrix of conditions, ranging from the admirable ones - such as prowess - through to others, considered less desirable - like disorders and dysfunctions - may in actual fact all is natural. They can only be the cumulative result of millions of years of continuous reproduction. A fluctuating 2:1 coefficient ratio at best, created generation within and after generation, establishing bespoke standards of a diverse global 'bilateral' gene pool through ever-changing combinations of diluting and/or strengthening chiral creation 'bilateral sway'. Though our TRUE bilateral profile is in essence largely invisible, it is no less real! Consider for example: Left or Right Handed? Left or Right eyed? Left or Right Eared? (For Information Purposes: there is also an automatic aspect at work and helping out elsewhere to varying degrees). Thinking in detail and in sequence or thinking creatively though appearing a little sporadic? Is there any evidence of cross or mixed dominance? These more neurological than physiological type questions may just be the easy ones as there could be many, many more, probably in increments of 23 or 46, depending on how it is viewed. (Rhetoric) Can you see a profile start to build? Can you see it in others? You see, it is invisible though it is there! But how balanced is it? True understanding \& appreciation may be the only medicine capable of and required to conclusively address and resolve many cases. Appropriate prudence in deciding which route to take, either the "make better people" route or staying firmly and purposefully committed to the other "make people better" route could be instrumental and would prove beneficial. Pleading ignorance as a third option could well have its very own route, although should prove to be the one least travelled. To pause for now, I hope to have struck a chord of curiosity with someone, somewhere and that somehow, 
together, we can consider this theory (inclusive) further with a view to better understand it and if called for, a goal to resolving it. Please consider: Practice and passion are every bit a qualification as permits and permission! Should you wish to, please circulate this theory as you see fit.

\section{Final Thought}

If researching, check out: "What are the causes of........ (select any condition listed below). Explanations are offered for some and a few appear not even to be considered though many are preceded by comments such as: 'the root causes are unknown', 'exactly what leads to ........ is not understood', 'the exact cause hasn't been identified yet' and so on. (This may take a moment or two or 25 years!) ${ }^{\star}$ It Doesn't Sense Make, that whilst many causes remain unknown, to judge some conditions as disorders or dysfunctions (an illness) when in actual fact they may be natural, albeit undesirable. This approach, though well-intentioned, could be misguided and have negative effects for the psychological wellbeing of those considered as sufferers by contrasting them with those bilaterally swayed toward the assumed, understandably, more favourable direction within the spectrum (wellness). It also introduces STIGMA, a quality that resides further on up that third route. Addictive behaviour/Anxiety disorders/ Asthma/Autism/Autoimmune disorders/ADHD/Bipolar disorder/ Natural Athleticism function*/certain cancers/Depression/Diabetes/ DYSLEXIA (including other co-morbid 'Dys-' family members)/ Eating disorders/Reliable Professional order ${ }^{\star} /$ Gender dysphoria (inclusive)/Migraines/Motor neurone disease/Multiple sclerosis/ Tinnitus/Tourettes/Obesity/Ordinary Fun People Syndrome* ${ }^{*}$ OCD/Paedophilia/Primitive reflexes (appreciation of these reflexes is of utmost importance)/PTSD/Schizophrenia/Sensory processing disorders/... This list in not exhaustive! $\left({ }^{*}\right.$ What about: NAf, RPo, OFPS? Do these not count, or are they simply ignored? They ARE "disorders"/“dysfunctions"!)Lastly and sadly (though the author 'wants' to be incorrect, but understands he may not be), 'Suicide behaviour disorder'. Proactively, purposefully and consciously swaying toward a 'solution', and quoting Socrates "the unconsidered life is not worth living", best way we start considering 'soonest!”).

BILATERAL SWAY - MANY MENTAL AND FUNCTIONAL CONDITIONS, GOOD AND NOT SO GOOD, COULD BE A DIRECT REFLECTION OF OUR TRUE FUNCTIONAL BILATERAL COHESIVE SYNTHESIS PROFILE.

Our whole working quality of both sides.

Thanks 'very much' for your time/consideration \& hope to hear your thoughts.

Best Regards

\section{EMMET McMANUS}

"For too long we have been battling this battle that won't be won,until there is an understanding; then it will be for everyone".

A personal message to 'my guys'. Despite many years hiding beneath the covers and studying my pain, nobody hides pain better than a person who is trying to remain sincere, for the ones that they love unconditionally. Sometimes the sincerest people are the ones who love beyond many challenges, Cry behind closed doors and fight battles that nobody knows about.

BE TRUE: Be consistent with fact and reality. 